\title{
Un estudio sobre el efecto de la figura en el razonamiento silogístico
}

\author{
J. A. García Madruga \\ Ice-Uned
}

\section{INTRODUCCION}

El estudio del razonamiento con silogismos categóricos ha despertado en la pasada década el interés de los investigadores. La característica fundamental de estos estudios recientes es el desarrollo de modelos que permiten definir de forma más precisa los problemas existentes y que han supuesto un importante avance en las investigaciones ( $G .^{a}$ Madruga, 1981). Otra caracteristica, que se ha revelado de gran interés, es el estudio de la influencia de la figura del si. logismo en el razonamiento de los sujetos.

El silogismo categórico es un tipo de argumento condicional formado por dos premisas y una conclusión. Tanto las premisas como la conclusión son enunciados en los que aparecen los cuantificadores (Todo» y "Algún), con sus negaciones "Ningún» y "Algún no». Tradicionalmente se han utilizado las letras mayúsculas, A, I, E y $O$ para caracterizar los enunciados que contienen estos cuantificadores:

A.- (Todo A es B)

I.- "Algún A es B»)

E.- "Ningún $A$ es $B$ »

O.- "Algún A no es B»
Universal afirmativa. Particular afirmativa. Universal negativa. Particular negativa.
Veamos un ejemplo:

Todo B es C

Algún A es B

Luego: Algún A es C

Las combinaciones de premisas y conclu. sión es lo que se llama modo del silogismo; el modo de nuestro ejemplo sería AI.I.

En la lógica clásica el sujeto de la conclu. sión está en la segunda premisa o premisa menor y el predicado en la primera o premisa mayor. En nuestro ejemplo, el sujeto de la conclusión es $\mathrm{A}$, el predicado $\mathrm{C}$, y el término medio $\mathrm{B}$.

Ahora bien, el orden de los términos en cada premisa puede variar de un silogismo a otro, con lo que nos da las cuatro figuras posibles:

\begin{tabular}{|c|c|c|c|}
\hline 1." Figura & 2.* Figura & 3." Figura & 4." Figura \\
\hline $\begin{array}{l}B-C \\
A-B\end{array}$ & $\begin{array}{l}C-B \\
A-B\end{array}$ & $\begin{array}{l}B-C \\
B-A\end{array}$ & $\begin{array}{l}C-B \\
B-A\end{array}$ \\
\hline$A-C$ & $A-C$ & $A-C$ & $A-C$ \\
\hline
\end{tabular}

Tradicionalmente los errores de los sujetos en el razonamiento silogístico han sido 
explicados mediante factores dependientes del modo del silogismo y no de la figura. Así, tanto la hipótesis del efecto catmósfe. ran (Woodworth y Seels, 1935; Sells, 1936), como las hipótesis de la conversión (Chapman y Chapman, 1959) y de las interpreta. ciones restringidas (Ceraso y Provitera, 1971) postulan la existencia de procesos de error generados por las características de las premisas del silogismo, independiente. mente de la disposición de los términos (figura).

El modelo de Erickson $(1974,1978)$ pos. tula la existencia de tres estadios: interpre. tación, combinación y elección de la forma verbal de la conclusión. Este modelo sostie. ne que, en cada uno de los tres estadios, los sujetos utilizan un tipo de representación semejante a los diagramas de Euler. La su. posición básica del modelo es que la causa de los errores de los sujetos es que realizan un análisis parcial e incompleto (Erickson, 1974, p. 308). La figura del silogismo es ig. norada en el modelo.

El modelo de Revlis (1975a y b) es una construcción compleja que sostiene que el razonamiento de los sujetos es totalmente lógico pero a partir de una interpretación errónea de las premisas. Su supuesto básico es que los sujetos convierten automática. mente todas las premisas del silogismo con lo que cambian la figura de éste. El modelo de Revlis no sólo ignora el efecto de la figu. ra en el razonamiento, sino que es radical. mente incapaz de explicarlo al postular la conversión automática.

\section{LOS ESTUDIOS SOBRE EL EF̀ECTO DE LA FIGURA DEL SILOGISMO.}

Los primeros estudios sobre la figura del silogismo fueron los de Frase (1968) y Pez. zoli y Frase (1968). Estos estudios fueron realizados, tratando de demostrar un isomorfismo entre las 4 figuras del silogismo y los 4 paradigmas de la mediación en tres estadios, usados en el aprendizaje de pares asociados. Las figuras 1, 2, 3 y 4 se corresponden con los paradigmas de encadenamiento hacia adelante, equivalencia de estí. mulo, equivalencia de respuesta y encade. namiento hacia atrás, respectivamente. Esta concepción teórica permitia predecir que el número de errores aumentaría progresivamente de la figura 1 a la 4. Los re. sultados de los estudios de Frase confirmaron la predicción al encontrar el menor nú. męro de errores en la figura 1 y el mayor en la figura 4 , aunque las diferencias entre las figuras 2 y 3 eran pequeñas. Roberge (1971) confirmó parcialmente estos resulta. dos al encontrar que la figura 4 producia el mayor número de errores, aunque los re sultados en la figura 1 no se adecuaban a las predicciones. No obstante, la analogía entre el razonamiento silogístico y el apren. dizaje de pares asociados no deja de ser una pura coincidencia sin significación real, ya que, en el mejor de los casos, sólo podría explicar las fuentes de error debidas a la fi. gura, dejando sin explicación las diferen. cias en la actuación de los sujetos según las distintas combinaciones de premisas (modo del silogismo).

Dickstein (1978) considera que el efecto de la figura sobre el razonamiento provie ne de que el (sujeto procesa la información de las premisas en dirección hacia adelante (de sujeto a predicado) o hacia atrás (de predicado a sujeto) influenciado por la direc. ción de la información presentada en las premisas) (1978a, p. 77).

En la figura $1 .\left(\begin{array}{ll}\mathrm{B} & \mathrm{C} \\ \mathrm{A} & \mathrm{B}\end{array}\right)$ los términos $\mathrm{A}$ y $\mathrm{C}$ están colocados de tal manera que el térmi. no $C$ ya realiza funciones de predicado y el término $\mathrm{A}$ de sujeto, existirá por tanto una tendencia a las conclusiones $\mathrm{A}$ - C que la si. logistica tradicional exige. Por el contrario, en la figura $4\left(\begin{array}{l}\mathrm{C} \cdot{ }_{\mathrm{B}}^{\mathrm{B}} \\ \mathrm{A}\end{array}\right)$ los términos $\mathrm{C}$ y $\mathrm{A}$ han cambiado su colocación respectiva haciendo de sujeto el término $\mathrm{C}$ y de predicado el término A, la tendencia será a conclu. siones de tipo $C$ - $A$, donde $C$ actúa de suje. to $y$ A de predicado. En las figuras 2 y 3

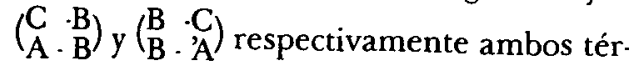


nos A y C realizan la misma función en las premisas (sujeto en la figura 2 y predicado en la 3) con lo que no habrá una tendencia especial en las conclusiones. En palabras de Dickstein «en la figura 1 ambas premisas tienen dirección hacia adelante y será pe. queña la tendencia en los sujetos a procesar la información de P. a S. En contraste, en la figura 4 ambas premisas proceden de $\mathrm{P}$ a $\mathrm{S}$ y aquí la tendencia a procesar la infor. mación en la dirección hacia atrás será máxima. Las figuras 2 y 3 serán intermedias ya que en ambas una premisa procede en dirección hacia adelante, mientras que la otra lo hace hacia atrás») (1978a, pp. 77-78).

Dickstein propone que los efectos de la figura se manifestarán en aquellos silogis. mos en que las conclusiones hacia adelan. te, es decir de S a P (A - C), son diferentes de las conclusiones hacia atrás, es decir de $\mathrm{P}$ a $\mathrm{S}(\mathrm{C} \cdot \mathrm{A})$. Dado que Dickstein en sus experimentos utiliza una tarea de evaluación, donde el sujeto sólo puede escoger las con. clusiones tradicionales hacia adelante (de $S$ a $\mathrm{P}$ ó $\mathrm{A} \cdot \mathrm{C}$ ), algunos sujetos extraerán correctamente las conclusiones hacia atrás (de P a S ó C - A) y erróneamente las aplica. rán a la dirección hacia adelante (de $S$ a $P$ ó A - C). Por tanto la hipótesis principal en Dickstein es que en aquellas combinaciones de premisas en que el procesamiento hacia adelante y hacia atrás llevan a diferentes conclusiones, la actuación mejor será en la figura 1, intermedia en las figuras 2 y 3 y peor en la figura 4. (1978a, p. 78).

Estas predicciones fueron confirmadas por los resultados de su experimento. La actuación de los sujetos fue significativamente mejor en la figura 1 , intermedia en las figuras 2 y 3 y peor en la figura 4 . La explica. ción de Dickstein sobre el efecto de la figura forma parte de su concepción general sobre los procesos de error en el razona. miento silogistico (1978b).

Johnson-Laird (1975; Johnson-Laird y Steedman, 1978) ha sido el primer investi. gados que ha elaborado un modelo de ra. zonamiento silogístico que incluye la influencia de la figura del silogismo. En el origen de este modelo están dos cambios teó. rico-metodológicos. En primer lugar, rom. pió con la tradición lógica, respetada hasta entonces por los psicólogos, que obligaba a que el sujeto de la conclusión estuviera siempre en la segunda de las premisas. En segundo lugar, y en relación con lo anterior, Johnson-Laird utilizó una tarea de construcción mediante la cual el sujeto ela. boraba su propia conclusión, sin estar limi. tado a las 5 alternativas de las tareas de se. lección.

En un primer experimento, utilizando sólo los silogismos con conclusión proposicional válida, encontraron un pronunciado efecto figural. Las figuras $1\left(\stackrel{B}{A} \cdot C_{B}\right)$ y 4 $\left(\begin{array}{c}C \\ B\end{array}-A_{A}\right)$ producían un sesgo especial a conclusiones A - C y C - A, respectivamente. En

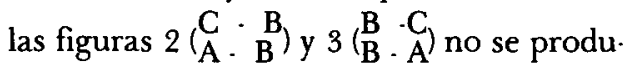
cían sesgos de respuesta significativas, aunque con una ligera tendencia A - C en la figura 2.

Estos resultados fueron confirmados en un segundo experimento (Johnson-Laird y Steedman, 1978). Además, en los silogismos con conclusión válida en sólo uno de los sentidos (A - C ó C - A) los sujetos la encon. traron fácilmente si coincidia con el efecto de la figura y dificilmente si no coincidía. Un claro ejemplo presentado por los autores $(1978, p .72)$ consiste en la comparación de los resultados en las combinaciones EI e IE en las figuras 1 y 4 . Asi, al ser la conclu sión compatible con la figura los problemas son más fáciles:

IE-4

EI-1

$\begin{array}{cc}\begin{array}{c}\text { Algún C es B } \\ \text { Ningún B es A }\end{array} & \begin{array}{c}\text { Ningún B es C } \\ \text { Algún A es B }\end{array} \\ \begin{array}{c}\text { Algún C no es A } \\ \text { (17 sujetos de 20) }\end{array} & \begin{array}{c}\text { Algún A no es C } \\ \text { (14 sujetos de 20) }\end{array}\end{array}$

mientras que al ser incompatible la conclusión con la figura los problemas se hacen más difíciles: 
IE-1

El-4

\begin{tabular}{cc}
$\begin{array}{c}\text { Algún B es C } \\
\text { Ningún A es B }\end{array}$ & $\begin{array}{c}\text { Ningún C es B } \\
\text { Algún B es A }\end{array}$ \\
\cline { 2 - 3 } $\begin{array}{c}\text { Algún C no es A } \\
\text { (5 sujetos) }\end{array}$ & $\begin{array}{c}\text { Algún A no es C } \\
\text { (8 sujetos) }\end{array}$ \\
\hline
\end{tabular}

Cuando el silogismo tenía conclusión vá lida en ambos sentidos, es decir dos conclusiones válidas, los sujetos tendian a escoger la solución compatible con el efecto de la figura. Aún en el caso de no existir conclu. sión válida el efecto figural se manifiesta en las conclusiones erróneas escogidas por los sujetos.

Para explicar los resultados encontrados, Johnson-Laird y Steedman (1978) han desarrollado un modelo analógico del razonamiento silogístico que incluye una explicación del efecto de la figura. Las dos caracte. rísticas principales del modelo en cuatro estadios propuesto, son la existencia de un proceso heuristico que genera una conclusión inicial y la existencia de unà cuarta fase de comprobación lógica de la conclusión inicial. de premisas EI que tienen conclusión válida sólo en la dirección A - C, es decir conclusión válida $\mathrm{O}$, EI-4 resultó significativamente más dificil que EI-1.

No obstante, el resultado más destacable fue la existencia de una tendencia de respuesta hacia conclusiones A - C en la combinación de premisas $\mathrm{AE} \cdot 2$, en la que no se predecia ninguna tendencia especial. Este hecho podria ser explicado mediante lo que podríamos llamar hipótesis fuerte del efecto figura. Según esta hipótesis en las combinaciones de premisas AE-EA, AI - IA y AO - OA, los sujetos realizan la in. ferencia partiendo de las premisas $\mathrm{E}, \mathrm{I}$ y $\mathrm{O}$, respectivamente, $y$ sustituyendo en esta premisa el término medio B por el otro tér. mino extremo situado en la premisa $A$. Esta hipótesis supone que el razonamiento, en estas combinaciones, está basado en una premisa-ancla y está favorecido por las in. terpretaciones de las premisas $\mathrm{A}$ en términos simétricos (A igual a B). Esta hipótesis fuerte predice los mismos efectos de figura que la hipótesis normal para las figuras 1 y 4, es decir conclusiones A - C y C - A, res pectivamente. Además predice:

a) Para AE-2, AI-2 y AO-2 conclusiones A.C (E, I y O respectivamente).

b) Para AE-3, AI-3 y AO-3 conclusiones C-A (E', I' y O' respectivamente).

c) Para EA-2, EA-2 y OA-2 conclusiones $C \cdot A$ (E', I' y O' respectivamente).

d) Para EA-3, IA.3 y OA.3 conclusiones A.C (E, I y O respectivamente).

La influencia de la figura del silogismo en la elección del sujeto y el predicado de la conclusión y su consiguiente influencia en la dificultad de los silogismos con con. clusión proposicional válida en un solo sen. tido (A - C ó C - A), ha sido confirmada en un primer experimento evolutivo (G.a $\mathrm{Ma}$. druga, 1981) cuyo objetivo principal era comprobar los procesos de error en el razo. namiento de los sujetos, comprobando al mismo tiempo las interpretaciones que realizaban. Así, encontramos que en los silo. gismos con conclusión válida en ambas di recciones, EA-1 y AI-1 había una tendencia a conclusiones A - C y en IA - 4 a conclusiones C - A. A simismo, en las combinaciones
En suma, la hipótesis del razonamiento ancla predice no sólo determinadas direc. ciones en la conclusión para las figuras 2 y 3 , sino también el tipo de conclusión coincidente con la premisa-ancla, independiente. mente de que sea válida o errónea.

\section{EXPERIMENTO}

\subsection{Planteamiento e hipótesis}

El objetivo principal del experimento fue la comprobación de un modelo general del razonamiento silogístico (G.a Madruga, 1981) que incluye una explicación del efecto figura. Se trataba de comprobar las pre. 
dicciones del modelo y, en particular, los efectos de las instrucciones diferenciales contra la verificación en la actuación de los sujetos. En cuanto al efecto de la figura el modelo sostiene dos hipótesis complementarias:

a) Hipótesis general: Existirá una tendencia a conclusiones A - C en la figura 1 y a conclusiones $\mathrm{C}$ - A en la figura 4 , no exis. tiendo tendencia especial para las figuras 2 y 3 .

b) Hipótesis razonamiento-ancla: En las combinaciones en que una de las premisas es universal afirmativa: AE, EA, AI, IA, $A O$ y $O A$, el razonamiento-ancla predice:

Conclusiones A.C, para AE-2, AI. 2 y AO-2

Conclusiones C-A, para AE-3, AI-3 y AO-3

Conclusiones C-A, para EA.2, IA. 2 y OA. 2

Conclusiones A.C, para EA.3, IA. 3 y OA. 3

\subsection{Método}

\subsubsection{Diseño y materiales}

El diseño experimental fue de covarian za con dos grupos. El grupo control recibió instrucciones normales y el grupo experimental las instrucciones contra la verificación. La covariable controlada fue inteligencia.

Cada grupo recibió un cuadernillo con unas instrucciones y una prueba de razonamiento (tarea de construcción) compuesta de 32 combinaciones de premisas, forman. do entre ambas pruebas el total de las 64 combinaciones de premisas posibles. Las dos pruebas son totalmente paralelas y de igual dificultad, con dos combinaciones por cada uno de los 16 modos del silogismo, y 8 combinaciones por cada una de las cuatro figuras. Para la covariable inteligencia se utilizó el (test) de factor (g)" de Cattell.

\subsubsection{Sujetos}

Los sujetos fueron 60 alumnos de C.O.U. del Instituto Nacional de Bachillerato "Car. denal Herrera Oria). Este centro puede ser considerado de clase media alta. Cada grupo contó con 30 sujetos, 15 de ellos varones y 15 mujeres (Grupo control; E.M. $=17$ años.8 meses; Grupo Experimental: E.M. $=17$ años -9 meses). La selección de sujetos fue aleatoria y todos ellos participaron de forma voluntaria.

\subsubsection{Procedimiento}

La prueba de razonamiento se pasó de forma individual a cada sujeto, controlan do la comprensión cabal de la tarea. El (test) de inteligencia se pasó de forma colectiva en grupos de un máximo de 20 suje. tos.

\subsection{Resultados}

Los resultados en cuanto a las dos hipótesis sobre el efecto de la figura, pueden observarse en la tabla 1. Tanto las predicciones de la hipótesis general como las del ra. zonamiento-ancla se cumplieron amplia. mente siendo significativas todas las tendencias de respuesta previstas (1).

\section{Discusión}

Los resultados, que confirman ampliamente ambas hipótesis, apuntan hacia una mayor tendencia a conclusiones $\mathrm{A} \cdot \mathrm{C}$ que C.A, en las figuras y combinaciones res. pectivas. Esto podría deberse a que el orden A - C es quizá más normal para los su. jetos en su experiencia cotidiana que el C - A, entre otras razones porque es precisamente el orden alfabético.

En cuanto al razonamiento-ancla, una cuestión que puede facilitar las tendencias de respuesta encontradas en las figuras 2 y 3 es que las conclusiones válidas en una sóla dirección ( $\mathrm{O}$ y $\mathrm{O}^{\prime}$ ) coinciden siempre con las tendencias que predice la hipótesis, 
Tabla 1

Porcentajes de conclusiones proposicionales $A-C$ y $C-A$ en ambos grupos, control y experimental y según las dos hipótesis sobre el efecto figura.

\begin{tabular}{|c|c|c|c|c|c|}
\hline & \multicolumn{2}{|c|}{ Grupo Control } & \multicolumn{2}{|c|}{ Grupo Experimental } \\
\hline & & $\begin{array}{c}\text { Conclusiones } \\
\text { A-C }\end{array}$ & $\begin{array}{c}\text { Conclusiones } \\
\text { C-A }\end{array}$ & $\begin{array}{c}\text { Conclusiones } \\
\text { A-C }\end{array}$ & $\begin{array}{c}\text { Conclusiones } \\
\text { C-A }\end{array}$ \\
\hline \multirow{4}{*}{ 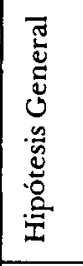 } & Fig. 1 & $77,78_{\mathrm{xxx}}$ & 22,22 & $82,44_{\mathrm{xxx}}$ & 17,56 \\
\hline & Fig. $\quad 2_{a}$ & 52,05 & 47,95 & 43,48 & 56,52 \\
\hline & Fig. $\quad 3 \mathrm{a}$ & 45,45 & 54,55 & 57,44 & 42,56 \\
\hline & Fig. $\quad 4$ & 27,68 & $72,32 \mathrm{xxx}$ & 24,33 & $75,67 \mathrm{xxx}$ \\
\hline \multirow{4}{*}{ 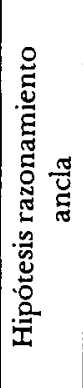 } & Fig. $\quad 1$ & $76,36 \mathrm{xxx}$ & 23,64 & $84,33_{\mathrm{xxx}}$ & 15,67 \\
\hline & Fig. $\quad 4$ & 22,43 & $77,57_{\mathrm{xxx}}$ & 24,40 & $75,60_{\mathrm{xx}}$ \\
\hline & {$\left[\begin{array}{c}\text { Ten- } \\
\text { dencia } \\
\mathrm{A}-\mathrm{C}_{\mathrm{b}}\end{array}\right.$} & $78,84_{\mathrm{xxx}}$ & 21,16 & $78,35_{\mathrm{xxx}}$ & 21,65 \\
\hline & $\begin{array}{c}\text { Ten- } \\
\text { dencia } \\
\text { C-A } b\end{array}$ & 27,73 & $72,27_{\mathrm{xxx}}$ & 35,19 & $64,81_{x}$ \\
\hline
\end{tabular}

a) En estas figuras no se incluyen las combinaciones de premisas donde actúa el razonamientoancla ya que se predicen efectos contradictorios dentro de una misma figura.

b) Según el razonamiento-ancla, las combinaciones de premisas con tendencia A.C son AO.2 y EA.3 en el grupo control y AE.2, AI-2, IA.3 y OA.3 en el grupo experimental. Las combinaciones de premisas con tendencias C.A son AE-3, AI-3, IA-2 y OA-2 en el grupo control y AO-3 y EA-2 en el experimental.

c) $\mathbf{x x x}=\mathrm{p}<, 001$

$\mathrm{xx}=\mathrm{p}<, 01$

$\mathrm{x}=\mathrm{p}<, 05$

aunque su influencia es pequeña (AO.2.O, AO-3.O', AE-3.O', EA-3.O, OA.2.O' $y$ OA.3.O).

En las hipótesis no se formuló la influencia de la figura en la dificultad del silogismo al favorecer una conclusión en una dirección coincidente $u$ opuesta a la única con. clusión válida. Esto no se hizo porque las comparaciones, tanto en el grupo experimental como en el control, eran entre figu. ras 1 y 4 por una parte y 2 y 3 por otra, con lo que los efectos son sólo parciales. No obstante, los resultados aunque generalmente no significativos, fueron acordes con la influencia de la figura (Grupo control: EI-4 más difícil que EI-2, IE-3 más difícil que IE-4; Grupo experimental: EI-3 más difícil que EI-1, IE-1 más difícil que IE-2).

Con respecto a la comparación con los resultados de otros estudios sólo es posible hacerlo con los de Johnson-Laird y Steedman (1978), ya que además de presentarlos 
en su totalidad, utilizaron también una ta. rea de construcción.

Como ya hemos analizado la hipótesis general fue comprobada por los propios autores. En cuanto a la hipótesis del razonamiento-ancla para las figuras 2 y 3 , el $64,19 \%$ de las conclusiones proporcionales coincidieron con la tendencia A - C y el $71,60 \%$ con la tendencia C - A. Estos resultados confirman claramente las predicciones aunque no se ha realizado una comprobación de la significación de las diferencias.

Quizá lo más importante de la hipótesis del razonamiento-ancla es que el estudio de los efectos de la figura del silogismo nos ha llevado a proponer un sistema heurístico concreto que utilizarían los sujetos en el ra. zonamiento. Por supuesto, en el origen está una analogía con los estudios desarrollados en el campo del razonamiento lineal, y, en particular, en las series de tres términos. Esto no es sorprendente ya que los proble. mas que plantea la influencia de los térmi. nos de las premisas en la conclusión, es de cir el efecto de la figura, son muy semejan. tes a los problemas planteados en las series de tres términos. Es más, la hipótesis general del efecto de la figura podria ser explicada mediante la teoría de Huttenlocher (1968, Huttenlocher y Weiner, 1971) sobre la importancia del sujeto gramatical en las tareas de razonamiento. En la figura $1\left(\begin{array}{c}\mathrm{B} \cdot \mathrm{C} \cdot \mathrm{B} \\ \mathrm{A} \cdot \mathrm{B}\end{array}\right.$ sólo el A es sujeto en la segunda premisa con lo que existiría tendencia a que lo fuera en la conclusión, es decir a conclusiones A - C. En la figura $4\left(\underset{B}{C} \cdot A^{-B}\right)$ es el término $C$ el que es sujeto de la primera premisa con lo que la tendencia seria a conclusiones C - A. En las otras dos figuras al ser los dos términos extremos bien sujetos, bien predicados, no existiria sesgo de figura.

Otra explicación alternativa provendría de la teoría desarrollada por Hunter (1957) también para las series de tres términos. Según esta teoría es la figura $4\left(\begin{array}{c}\mathrm{C} \cdot \mathrm{B} \\ \mathrm{B} \cdot \mathrm{A}\end{array}\right)$ el suje. to codifica la primera premisa $\mathrm{y}$ añade la segunda, C.B....B.A, con el resultado de un sesgo hacia las conclusiones C.A. En la primera figura $(\stackrel{\mathrm{B} \cdot \mathrm{C} \cdot \mathrm{B}}{\mathrm{A}})$ sería algo más complejo pues habría que codificar primero la segun. da premisa: A.B....B.C: A.C. En las otras dos figuras no existiria, en principio, ningún sesgo en sus conclusiones.

En suma, podemos concluir que la hipó. tesis del razonamiento-ancla, cuyas predic. ciones se han visto confirmadas, supone un avance en el estudio del razonamiento silogístico ya que las tendencias de respuesta encontradas obligan a postular un determinado proceso en el razonamiento de los sujetos. Por supuesto, sería necesario ampliar y corroborar estos resultados con nuevos estudios sobre el problema. 


\section{Referencias}

Ceraso, J. y Provitera, A. "Sources or error in Syllogistic reasoning». Cognitive Psychology, 1971, 2, 400.4 10. Trad. española en Delval, J. A. (ed.) Investigaciones sobre lógica y psicología. Alianza Universidad, Madrid, 1977.

Chapman, L. J. y Chapman, J. P. (Atomosphere effect reexamined). Journal or Experimental Psychology, 1959, 58, 220.226. Trad. española en Delval, J. A. (ed.) Investigaciones sobre lógica y Psicología Alian. za Universidad, Madrid, 1977.

DisckTEIN, L. S. "The effect of figure in syllogistic reasoning». Memory and Cognition, 1978, 6, 76-83.

DiCKSTEIN. L. S. (Error processes in syllogistic reasoning». Memory and Cognition, 1978, 6, 537-543.

ERICKSON.J. R. "A set analysis theory of behavior in formal syllogistic reasoning tasks». En Solso, R. L. (ed.) Theories of cognitive psychology: The Loyola Symposium, 1974. Hillsdale, N. J., Erlbaum.

ERICKSON: J. R. «Research on Syllogistic Reasoning». En R. Revlin y R. Mayer (eds.) Human Reasoning, 1978, Washington, D. C., Winston.

FRASE L. T. (Associative factors in syllogistic reasoning». Journal of Experimental Psychology, 1968, 76, $407 \cdot 412$.

Garcia MADRLGA. J. A. Modelos de razonamiento silogístico. Tesis doctoral no publicada. Universidad Complutense, Madrid, 1981.

HUNTER, I. M. L. (The solving of three term series problems». British Journal of Psychology," 1957, 48, 286-298.

HUTTENLOCHER, J. “Constructing spatial images, a strategy in reasoning». Psychological Review, 1968, $75,550-60$.

HUTTENLOCHER, J. Y WEINER, S. «Comprehension of instructions in variyng contexts». Cognitive Psycho$\log y, 1971,2,369-385$.

Johnson.LAIRD, P. N. «Models of deduction». En R. J. Falmagne (ed.) Reasoning: Representation and Process. 1975. Hillsdale, N. J., Erlbaum.

Johnson.Laird, P. N. Y SteEdMaN. M. "The Psychology of Syllogisms). Cognitive Psychology, 1978, 10, 64.99.

Pezzoli, J. A. Y FRASE. L. T. «Mediated facilitation of syllogistic reasoningw. Journal of Esperimental Psychology, 1968, 78, 228.232.

REVLIS, R. ((Syllogistic reasoning: Logical decisions from a complex data base). En R. Falmagne (ed.): Reasoning: Representation and Process. 1975, Hillsdale, N. J., Erlbaum.

REVLIS, R. "Two models of syllogistic reasoning: Feature selection and conversion». Journal of Verbal Learning and Verbal Behavior, 1975, 14, 180-195.

ROBERGE, J. J. «Further examination of mediated associations in deductive reasoning». Journal of Experimental Psychology, 1971, 87, 127.129.

SELLS, S. B. "The atmosphere effect: an experimental study of reasoning». Archives of Psychology, 1936, $29,3.72$.

WOODWORTH, R. S. y Sells, S. B. "An atmosphere effect in formal syllogistic reasoning". Journal or Experimental Psychology, 1935, 18, 451-460. Trad. castellana en J. A. Delval (ed.): Investigaciones sobre lógica y psicología, Alianza, Madrid, 1977.

\section{Nota}

(1) Una presentación completa de los resultados, asi como una descripción más precisa de todo el experimento puede verse en García Madruga (1981). 


\section{Resumen}

Los estudios sobre el razonamiento con silogismos categóricos han demostrado que la disposición de los términos de las premisas en las figuras 1 y 4 , influye en la elección del sujeto y el predicado de la conclusión y, por tanto, afecta a la dificultad del silogismo. El objetivo de este estudio fue comprobar, además de la hipótesis general, la hipótesis del arazonamiento-anclas. Esta hipótesis precide en las combinaciones donde una de las premisas es universal afirmativa ( $A E, E A, A I, I A, A O$ y $O A$ ), la existencia también de un efecto figural en las figuras 2 y 3. Ambas hipótesis fueron comprobadas dentro de un diseño de covarianza con dos grupos, realizado para contrastar un modelo general del razonamiento silogístico. Los sujetos fueron 60 alumnos de C.O.U., cada uno de ellos recibió un cuadernillo con 32 combinaciones de premisas, formando entre los dos grupos el total de 64 combinaciones posibles. Los resultados se compararon con los de otros autores y se discutieron en el contexto de sus semejanzas con algunos estudios de las series de tres términos.

\section{Résumé}

Les études sur le taisonnement avec des syllogismes catégoriques ont démontré que la disposition des termes des prémises dans les figures 1 et 4 , influence sur le choix de sujet et le prédicat de la conclusion, et dond, elle affecte la dificulté du syllogisme. L'objetif de cet étude a été de prouver en plus de l'hypothèse generale, l'hypothèse du araisonament-ancres. Cette hypothèse predit, dans les combinaisons où une des prémisses est universelle affirmative ( $A E, E A, A I, I A, A O$ et $O A$ ) l'existence aussi d'un effet relatif aux figures 2 et 3. Les deux hypothèses ont été vefifiés dans un dessin de covariance avec deux groups, réalisé pour contraster un modèle general du raisonement syllogistique. On a choisi 60 élèves de C.O.U., dont chacun d'eux a reşu un petit cahier avec 32 combinaisons de prémises, formant entre les deux groupes le total de 64 combinaisons posibles. Les résultats confirmèrent les prédictions des deux hypothéses. Ces résultats ont été comparé avec ceux d'autres auteurs et ils se discuterent dans le contexte de ses ressemblances avec quelques études des series de 3 termes:

\section{Summary}

Previous studies about categorical syllogistic reasoning have shown that disposition of terms in the premises, in figures 1 and 4, affects the election of subject and predicate of conclusion and, then, it also affects the difficulty of syllogism. Apart the general hypothesis, the aim of this paper was to test the hypothesis of ranchor-reasonings. That hypothesis predicts that when there is a universal affirmative premise ( $A E, E A, A I, L A, A O, O A)$ there will be a figural effect on figures 2 and 3 . A design of covariance with two groups was used to test a general model of syllogistic reasoning: both hypotheses were tested as valid. Subjects were 60 college students, each of them was given a pamphlet with 32 combinations of premises. Thert were two groups and 64 possible combinations. Results support predictions of both hypotheses. There results are compared with other studies and are discused in relation with some similar studies about three-term series problems. 